\title{
Role of Macrocyclic Conformational Steering in a Kinetic Route toward Bielschowskysin
}

\author{
Paul D. Scesa, Lyndon. M. West*, and Stéphane P. Roche* \\ Department of Chemistry and Biochemistry, Florida Atlantic University, Boca Raton, Florida 33431, United States. \\ Supporting Information Placeholder
}

\begin{abstract}
Macrocyclic furanobutenolide-derived cembranoids (FBCs) are the biosynthetic precursors to a wide variety of highly congested and oxygenated polycyclic (nor)diterpenes (e.g. plumarellide, verrillin or bielschowskysin). These architecturally complex metabolites are thought to originate from site-selective oxidation of the macrocycles' backbone and a series of intricate transannular reactions. Yet the development of a common biomimetic route has been hampered by a lack of synthetic methods for the pivotal furan dearomatization in a regio- and stereoselective manner. To address these shortcomings, a concise strategy of chemo- and stereoselective epoxidation followed by a kinetically-controlled furan dearomatization is reported. The surprising switch of facial $\alpha$ : $\beta$-discrimination observed in the epoxidations of the most strained $E$-acerosolide versus $E$-deoxypukalide and $E$-bipinnatin $\mathrm{J}$ derived macrocycles has been rationalized by the $3 \mathrm{D}$-conformational preferences of the macrocyclic scaffolds. A careful conformational analysis of these macrocycles by VT-NMR, and NOESY experiments at low temperature was supported by DFT calculations to
\end{abstract} characterize these equilibrating macrocyclic conformers. The shift of conformational topology associated with a swing of the butenolide ring in $E$ deoxypukalide is in general agreement with the reversal of $\beta$-selectivity observed in the epoxidation. We also describe the downstream functionalization of FBCmacrocycles, and how the $\mathrm{C}-7$ epoxide configuration is retentively translated to the C-3 stereogenicity in dearomatized products under kinetic control to secure the requisite $(3 S, 7 S, 8 S)$-configurations for the bielschowskysin synthesis. Unlike previously speculated, our results suggest that the most strained FBCmacrocycles bearing a $E-\left(\Delta^{7,8}\right)$-alkene moiety may stand as the true biosynthetic precursors to bielschowskysin

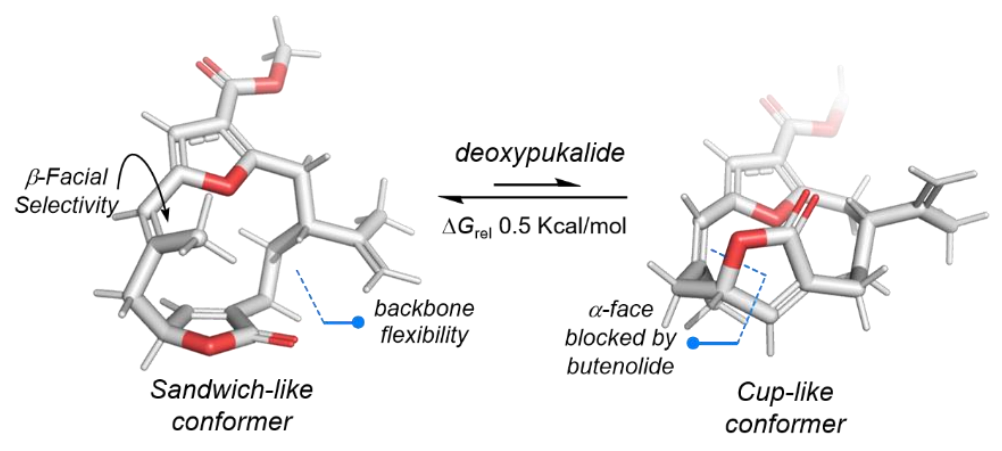
and several other polycyclic natural products of this class.

Conformationally Guided Selectivity

Introduction. The furanobutenolide-derived cembranoids (FBCs) from gorgonian corals ${ }^{1}$ constitute a large family of highly oxygenated and stereochemically complex polycyclic diterpenes possessing a broad range of biological activities. ${ }^{2}$ Unlike other terpenes, FBCs have a unique feature in that they are built upon the functionalization of a parent 14membered ring macrocyclic skeleton. Typically, the polycyclic carbon skeleton of terpenes such as taxanes or ent-kauranes are forged by specialized terpene cyclase enzymes. A hallmark of these enzymes is the conformationally-controlled formation of carbon-carbon (C-C) bonds from acyclic terpenyl precursors in a regio- and stereoselective manner to generate structural diversity in (poly)cyclic skeletons (cyclase phase) $)^{3,4}$ Further molecular complexity is often added by oxidase enzymes at a later stage by installing chemo- and stereoselectively key oxygenated functionalities at specific positions of the (poly)cyclic scaffolds (oxidase phase). ${ }^{5}$ For the macrocyclic FBCs, like for most (mero)terpenoid skeletons, a biosynthetic cyclase gene or enzyme has not yet been identified. ${ }^{6}$ In many instances, it is likely that no dedicated terpene-cyclase enzymes exist for the

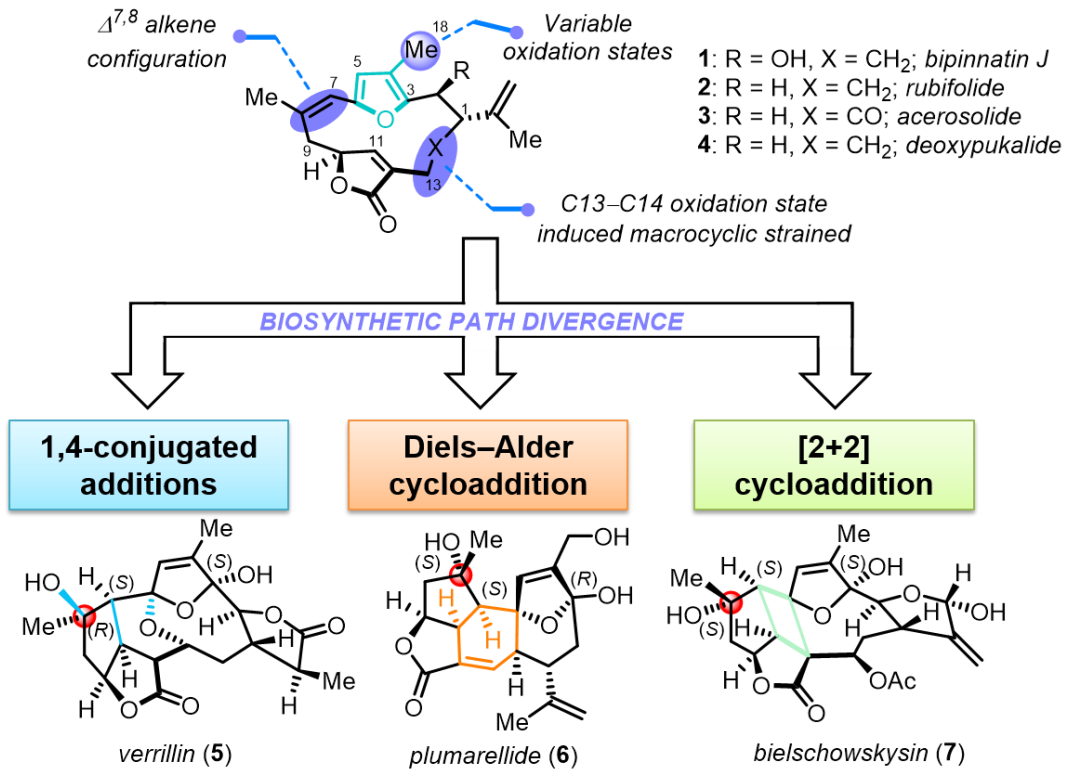

Figure 1. Unified biosynthetic proposal highlighting various tactical transannular reactions originating from $\mathrm{FBC}$-macrocycles 1-4, and the divergence of $\mathrm{C}-3 / \mathrm{C}-8$ relative and absolute configurations.

key $\mathrm{C}-\mathrm{C}$ bond-forming steps. ${ }^{7}$ Instead, an oxidase enzyme promoted pathway is at play to trigger either an oxidative cyclization cascade, or the generation of higher oxidation state 
metabolites which -under specific conditions (i.e. Lewis/Brønsted acidic or photochemical) - may rearrange to create the needed polycyclic scaffolds. As shown in Figure 1, some of the most complex cembranoid metabolites such as verrilin (5), plumarellide (6), and bielschowskysin (7) have been proposed to derive from a series of relatively challenging transannular reactions such as a 1,4-conjugated addition, a Diels-Alder, or a [2+2] cycloaddition. ${ }^{2,8}$ Even if the common biosynthetic origin of these natural products 5-7 remains speculative, ${ }^{2}$ it is unlikely that a cyclase enzyme is involved in forming the various polycyclic scaffolds. Indeed, these complex natural products have been postulated to originate from lower oxidation-state metabolic precursors (e.g. Z-bipinnatin $\mathbf{J}(\mathbf{1})$ or Z-rubifolide (2)). ${ }^{2}$ Strickingly, $E-\left(\Delta^{7,8}\right)$-configured FBCmacrocycles are rare in nature, while their corresponding trans $(7 S, 8 R)$-epoxides are promiscuous (e.g. pukalide, lophotoxin), thus nourishing the idea that the more flexible macrocyclic $Z$ isomers and their related cis epoxides might represent the biosynthetic precursors of numerous polycyclic natural products of this class. ${ }^{2 b}$ In contrast to this idea, earlier studies pointed out a lack of success in the chemo- and stereocontrolled epoxidation of Z-FBC-macrocycles. ${ }^{9}$ Therefore, we hypothesized that an oxidative furan dearomatization prompted by a stereoselective $E$ - $\left(\Delta^{7,8}\right)$-alkene oxidation might reveal a more realistic biomimetic path (Figure 1). ${ }^{10}$ Given the current lack of data on the stereocontrolled formation of $\mathrm{C}-7 / \mathrm{C}-8$ epoxide diastereomers, ${ }^{11}$ and on the regioselective oxirane opening, the overall mechanistic dearomatization process of FBC-macrocycles remains elusive. ${ }^{12}$ To address these shortcomings, the role played by the $\mathrm{C}-18$ oxidation state in tuning the furan's reactivity toward dearomatization is discussed herein, ${ }^{13}$ as well as the control of the C-8 tertiary hydroxyl absolute configuration. The impact of different oxidation levels at $\mathrm{C}-13 / \mathrm{C}-14$ on the macrocycles strain was evaluated, ${ }^{14}$ to shed a new light on the importance of macrocyclic conformational topology towards reactivity. We report here a surprising switch of stereoselectivity for the trans epoxides obtained from kinetic epoxidations of $E$-configured $\left(\Delta^{7,8}\right)$-FBC macrocycles. Along these lines, the ensuing dearomatization stereoselectivity outcome was also evaluated under kinetic and thermodynamic conditions. By taking advantage of the macrocyclic conformational bias at low temperature, a synthetic sequence toward bielschowskysin was established with a pivotal regio- and stereoselective furan dearomatization triggered by epoxide ring opening with full control over the newly formed $(3 S, 7 S, 8 S)$-stereogenic centers. Despite several elegant and innovative synthetic approaches toward bielschowskysin, ${ }^{15}$ only Nicolaou ${ }^{16}$ and us ${ }^{10}$ were able to build the intricate tricyclo[9.3.0.0]tetradecane architecture of the natural product. This study finally informs about the conformational dynamics of FBC-macrocycles, and the role of intertwined strategical oxidations and chemoselective $\mathrm{C}-\mathrm{C}$ bond-forming steps in the biosynthetic pathways of polycyclic FBCs.

\section{Results and Discussion.}

1. Conformational macrocyclic constraints on the $\Delta^{7,8}$ alkene $E / Z$-photoisomerization outcome and the epoxidation stereocontrol.

Our initial study started with the isolation of $E$-acerosolide (3) from Pseudopterogorgia acerosa in adequate quantities (ca. $500 \mathrm{mg}$ ) to investigate the key steps of epoxidation and furan dearomatization. Given that $E \mathbf{- 3}$ is a singular example of the 14membered FBC-macrocycles found in nature harboring a $\Delta^{7,8}$ olefin of $E$-configuration, this metabolite was an exciting starting point to study the stereochemical outcome of our synthetic approach (Scheme 1). ${ }^{10}$ The underlying hypothesis for the study of $E$-3's reactivity was based on the fact that a methyl ester group at $\mathrm{C}-18$ might be critical to tame the furan oxidation and generate the pivotal dearomatized exo-enol ether ketal required en route to bielschowskysin. After extensive conditions' optimization, the trans $(7 S, 8 R)$-epoxide 8a was obtained as the major diastereomer from the epoxidation of $E$ 3 in $96 \%$ yield (brsm). This epoxidation arising from the $\alpha$-face

Scheme 1. Photochemical E/Z-isomerizations and the resulting stereochemical outcome of the $\Delta^{7,8}$ olefin facial epoxidations ${ }^{a, b}$

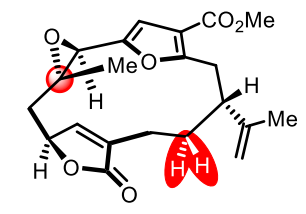

(7S,8R)-9a : (7R,8S)-9b

$1: 10$

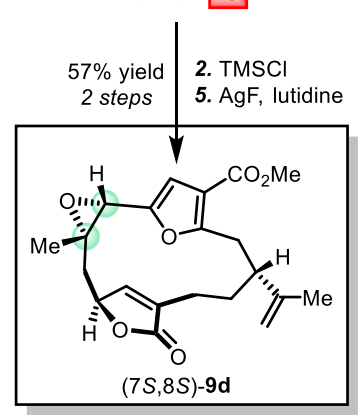

Desired diastereomer

with adequate configurations at C-7/C-8
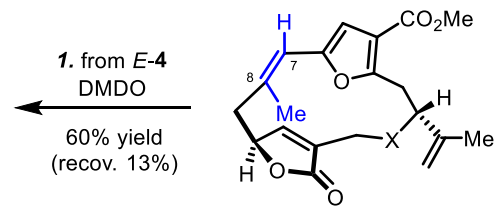

E-acerosolide (3), $\mathbf{X}=\mathrm{CO}$

E-deoxypukalide (4), $\mathrm{X}=\mathrm{CH}_{2}$
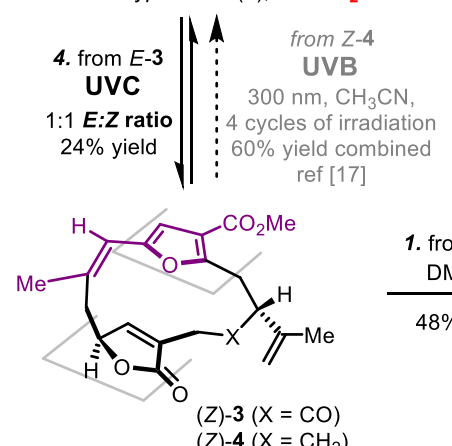

$(Z)-3(X=C O)$
$(Z)-4\left(X=\mathrm{CH}_{2}\right)$
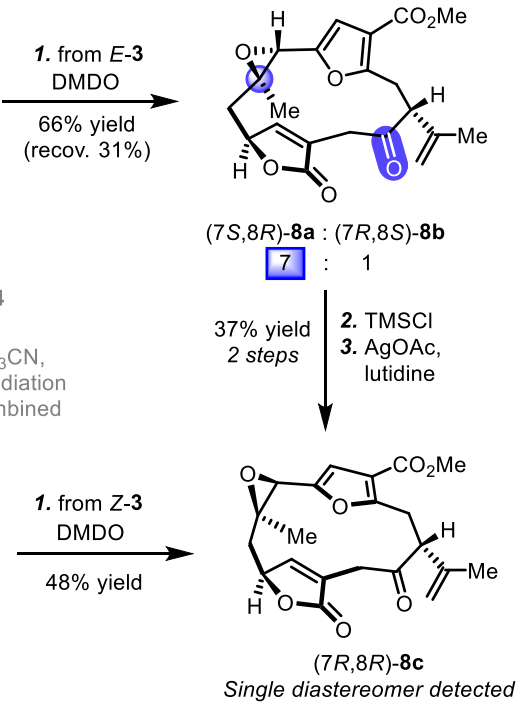

${ }^{a}$ Reagents and conditions: (1) DMDO (1.0-2.0 eq.), acetone (50-75 mM), $0{ }^{\circ} \mathrm{C}, 1-2 \mathrm{~h}$; from $Z$-3 a $1.7: 1$ product ratio of epoxide 8c and bisepoxide SI-2 was obtained. (2) TMSCl (1.1 eq.), $0{ }^{\circ} \mathrm{C}, \mathrm{CH}_{2} \mathrm{Cl}_{2}$ (13 mM), 1-2 h. (3) $\mathrm{AgOAc}$ (1.1 eq.), lutidine (1.1 eq.), $\mathrm{CH}{ }_{2} \mathrm{Cl} 2$, - 78 ' $\mathrm{C}, 2-3$ h. (4) $\operatorname{UVC}(\lambda=254 \mathrm{~nm}), \mathrm{CH}_{3} \mathrm{CN}, \mathrm{rt}, 2 \mathrm{~h}, E / Z$-photostationary state (1:1 ratio). (5) $\mathrm{AgF}$ (5.2 eq.), lutidine (1.9 eq.), toluene (63 mM), $0{ }^{\circ} \mathrm{C}, 0.5$ h.

${ }^{b}$ Only major diastereomers drawn in each reaction for simplicity. 
of the $\Delta^{7,8}$ alkene was highly stereoselective but generated the tertiary stereocenter with the undesired $(8 R)$-configuration towards 7 . Therefore, we selected to evaluate the epoxidation of $Z$-3. The $Z / E$-isomerization of $Z$-deoxypukalide (4) and other $Z$-FBC-macrocycles under UVB conditions was reported to be a challenging and capricious reaction (vide infra), seemingly the reverse $E / Z$-alkene isomerization has never been documented. ${ }^{17} \mathrm{~A}$ side-by-side UVB photoirradiation of $Z-3$ and $E-3$ for 2 hours in separate reaction flasks revealed that both compounds isomerized to afford a 2:1 E/Z-ratio characteristic of a photostationary state. Remarkably, a UVC irradiation $(\lambda<$ $260 \mathrm{~nm}$ ) of an acetonitrile solution of $E-3$ in a Quartz reaction vessel formed $Z-3$ preferentially although remaining in a photostationary state equilibrium with $E-3$ (1:1 E/Z-ratio). Both isomers were easily separated and isolated by preparative thinlayer chromatography albeit in a low $24 \%$ overall yield. ${ }^{18} \mathrm{Z}-3$ was then engaged in epoxidation with DMDO, but the $(7 R, 8 R)$ cis epoxide $8 \mathbf{c}$ was the sole diastereomer formed in the reaction, as expected for an epoxidation arising selectively from the macrocycle's $\beta$-face. ${ }^{9}$ The configuration of both $\mathrm{C}-7$ and $\mathrm{C}-8$ stereocenters was confirmed through another synthetic route starting from the $(7 S, 8 R)$-epoxide 8a. The stereospecific epoxide opening of $\mathbf{8 a}$ to the corresponding $(7 S, 8 R)$ chlorohydrin was achieved with stereoretention at C-7, and an inversion of configuration occurred during the re-cyclization of the oxirane moiety mediated by AgOAc to afford another sample of 8c (Scheme 1). These results confirm that the epoxidation of Z-3, like for other Z-FBC-macrocycles is likely controlled by the steric hindrance staged by their innate strained-conformation. Presumably, FBC-macrocycles bearing a Z-configured olefin fold preferentially into a sandwich-like conformation in which the furan and butenolide rings are relatively parallel to another, thus blocking any reagent to approach from the concave $\alpha$-face. ${ }^{19}$ This principle of peripheral attack from the $\beta$-face of $Z$-FBC macrocycles appears to be general due to the steric crowding from the butenolide nearby the double bond. ${ }^{20}$ In fine, epoxidation of $Z$ FBC macrocycles (e.g. bipinnatin J (1), rubifolide (2)) can therefore only deliver products of $(7 R, 8 R)$-configurations which cannot be exploited toward stereoselective dearomatization, and the synthesis of any of the natural products 5-7 shown in Figure 1. At this stage of the study, the question of C-8 stereocontrol remained unanswered.

Hence, we turned back our attention to the epoxidation of the $E$-configured FBC-macrocycles $E-\mathbf{4}$ and $E-10$ (Schemes 1-2). $E$-3, $E$-4, $E$-isopukalide ${ }^{13}$, and $E$-acerolide ${ }^{21}$ are the only four isolated metabolites presenting the $E$-configured $\Delta^{7,8}$ olefin. Epoxidation of $E-\mathbf{4}$ proved to be relatively high yielding by providing the trans epoxide $(7 R, 8 S)-9 b$ in $60 \%$ yield with an unexpected 10:1 diastereomeric ratio (Scheme 1). Of importance, the minor $(7 S, 8 R)$-epoxide diastereomer 9a was separated and isolated to provide a pure synthetic sample of (+)pukalide which completed the first asymmetric synthesis of this natural product. $^{11,22}$ A 2-step sequence of oxirane opening/closing from $9 b$ with TMSCl followed by AgF resulted in the isolation of epoxide 9d (57\% overall yield) possessing the desired $(7 S, 8 S)$-configuration en route to bielschowskysin. The switch of diastereoselectivity in the epoxidation of $E-4 \mathrm{vs}$ $E-3$ appeared to be related to the C-14 functionalization (methylene vs carbonyl). To rationalize this result, we hypothesized that either an electrostatic effect from the carbonyl might force the facial epoxidation selectivity, or that a change in macrocyclic strain might result in a subtle conformational (de)stabilization hence the facial discrimination. As eluded above, $E$-FBCs such as $E$ deoxypukalide (4) are typically unstable which explains why their chemistry has been relatively underexplored. $E$-configured FBC macrocycles usually present characteristic line broadening of numerous peaks across their ${ }^{1} \mathrm{H}$ NMR spectra at room temperature $(293 \mathrm{~K})$. This effect is consistent with the presence of several populations of conformers in equilibrium and rapidly interconverting over the NMR time-scale of the ${ }^{1} \mathrm{H}$ experiment. Therefore, a variable-temperature NMR (VT-NMR) experiment from -60 to $55^{\circ} \mathrm{C}$ was undertaken to evaluate possible changes of conformations in E-4 (Figure 2). ${ }^{18}$ Upon sample heating during spectral acquisition several peaks sharpened, and a resolved set of three multiplets appeared at $328 \mathrm{~K}$ between 2.15 and $2.65 \mathrm{ppm}\left[\delta_{\mathrm{H}} 2.58(\mathrm{brd}, J=13 \mathrm{~Hz}, 1 \mathrm{H})\right.$; 2.39 (br t, $J=13 \mathrm{~Hz}, 1 \mathrm{H}$ ); 2.22 (br dd, $J=13$ and $6 \mathrm{~Hz}, 1 \mathrm{H})]$. Below $0{ }^{\circ} \mathrm{C}$ (temperature of coalescence: $T_{C}$ ), two distinct sandwich-like conformers were observed in a $2: 1$ ratio. The spectrum collected at $T_{C}(273 \mathrm{~K})$ presented resonances with an asymmetric line broadening in the downfield region for $\mathrm{H}-5$ and $\mathrm{H}-11$, while $\mathrm{H}-7$ and $\mathrm{H}-10$ appeared as split resonance bands $\left(\delta_{\mathrm{H}-7} 5.92 / 6.02 \mathrm{ppm}\right.$ and $\delta_{\mathrm{H}-10} 5.24 / 5.18 \mathrm{ppm}$ for the major and minor conformers respectively). The resolved proton resonances were used to determine the ratio of conformers and the equilibrium constant $K_{\text {eq }}=1.92\left(\Delta G^{\circ}=0.303 \mathrm{Kcal}_{\mathrm{mol}}{ }^{-1}\right)$. Furthermore, the activation energy barrier, $\Delta G^{\ddagger}$, for the

Figure 2. A. Conformational and stereochemical assignments of the major $E-\mathbf{4}^{\prime}$ and minor $E-\mathbf{4}$ conformers of deoxypukalide from NOESY correlations across the furanocembranoid macrocycles at $40{ }^{\circ} \mathrm{C} .{ }^{a} \mathbf{B}$. Stereochemical models of epoxidation for $E$-acerosolide (3) vs $E$-deoxypukalide (4) based on experimental thermodynamic and kinetic parameters obtained by VT-NMR.
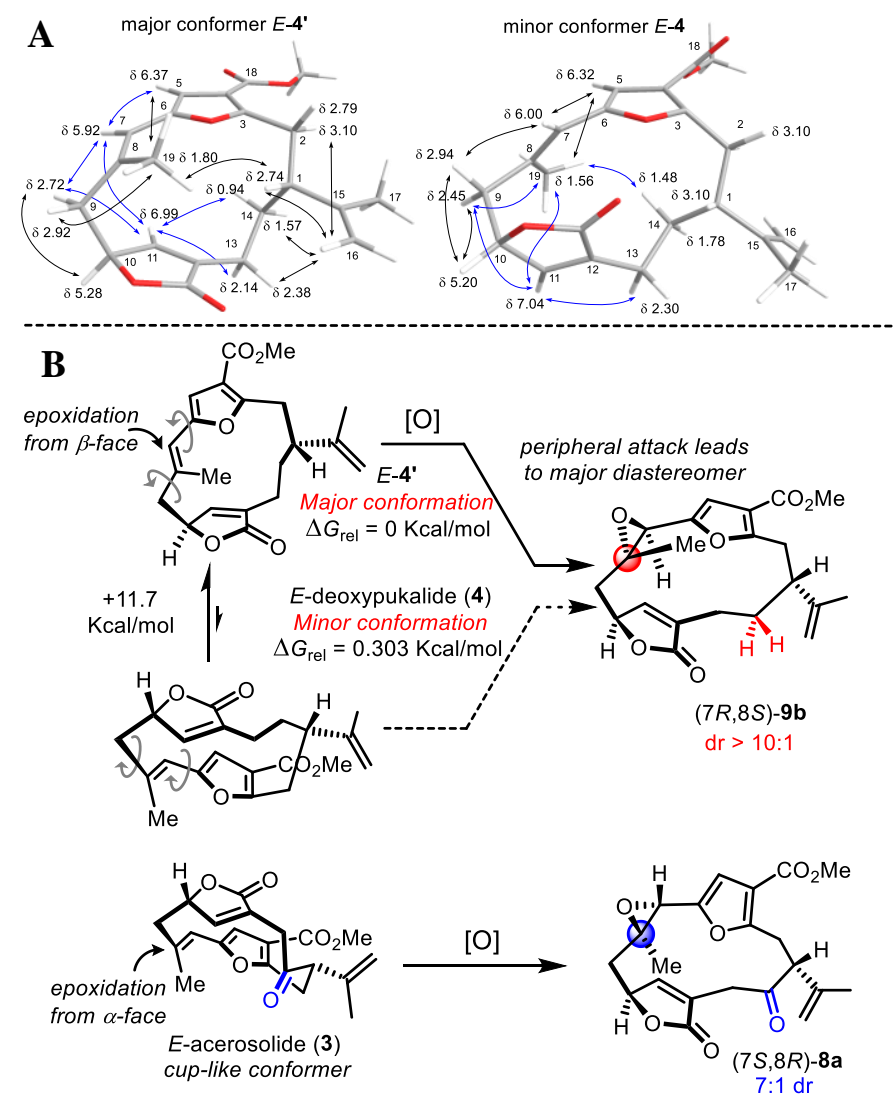

${ }^{a}$ Key NOESY correlations securing the determination of the FBCmacrocycles main conformation and $\alpha$-face (highlighted in blue). 
conformational exchange was calculated at $\mathrm{T}=233 \mathrm{~K}$ (temperature of best spectral resolution) from the coalescence temperature $T_{c}$ (approximately $273 \mathrm{~K}$ ) as follows; Where $a=$ $4.575 \times 10^{-3}$

$$
\Delta G^{\ddagger}=a T\left(9.972+\log \frac{T_{c}}{\Delta v}\right)=11.7 \mathrm{kcal} \cdot \mathrm{mol}^{-1}
$$

and $\Delta v$ is the difference in frequency $(\mathrm{Hz})$ from a pair of resolved but exchanging protons $(25.6 \mathrm{~Hz}$ for $\mathrm{H}-11$ at $233 \mathrm{~K})$. With the kinetic and thermodynamic parameters of conformational exchange determined, we set out to further elucidate the structural differences for the two conformers. The spectral assignment of proton chemical shifts were obtained from ${ }^{1} \mathrm{H}-{ }^{1} \mathrm{H}$ COSY, HSQC and $\mathrm{HMBC}$, while a detailed conformational analysis was conducted using the NOESY spectrum at $-40{ }^{\circ} \mathrm{C}$ or $233 \mathrm{~K}$ (Figure $2 \mathrm{~A}$ ). ${ }^{18}$ A preliminary survey of the NOESY spectrum revealed a wealth of cross-peaks with positive phase attributed to nuclear Overhauser effect interactions (NOESY-type correlations) as well as many crosspeaks with negative phase attributed to chemical exchange (EXSY-type correlations). These data unequivocally confirm the observation of conformers interconverting on the NMR time scale as well as supporting the proton chemical shift assignments. In the major conformer $\left(E-4^{\prime}\right.$ : novel and more open sandwich-like conformation), NOESY cross-peaks from H-7 to H-5, H-9 $\alpha$ and $\mathrm{H}-11$ as well as from $\mathrm{H}-11$ to $\mathrm{H}-9 \alpha, \mathrm{H}-$ $13 \alpha$ and $H-14 \alpha$ indicated these protons were present on the $\alpha$ face of the molecule. On the other hand, cross-peaks from $\mathrm{H}_{3}$ 19 to $\mathrm{H}-9 \beta$ and $\mathrm{H}-1$ indicated these protons were on the $\beta$-face of the molecule. In the minor conformer $[E-4$ : similar conformation to $E$-acerosolide (3)], NOESY cross-peaks from $\mathrm{H}-11$ to $\mathrm{H}-9 \alpha, \mathrm{H}-13 \alpha$ and $\mathrm{H}_{3}-19$ as well as from $\mathrm{H}_{3}-19$ to $\mathrm{H}-9 \alpha$, $\mathrm{H}-14 \alpha$ indicated these protons were present upon the $\alpha$-face of the molecule, while cross-peaks from $\mathrm{H}-7$ to $\mathrm{H}-9 \beta$, and from $\mathrm{H}$ 1 to $\mathrm{H}-13 \beta$ indicated these protons were on the $\beta$-face. Taken together, these spectroscopic data confirm that the major conformer population observed below $0{ }^{\circ} \mathrm{C}$ for deoxypukalide $E-4^{\prime}$ exhibits a different folding conformation than $E-3$ with a swing of the butenolide ring below the median plane of the macrocycle. In this more open sandwich-like conformation, the butenolide moiety is now hindering the $\alpha$-face, therefore forcing the stereoselective peripheral attack of DMDO from the $\beta$-face to obtain the $(7 S, 8 R)$-stereoisomer $9 \mathrm{c}$ quasi exclusively.

The stereochemical model proposed in Figure $2 \mathrm{~B}$ rationalizes for the first time the $\alpha: \beta$ selectivity in the epoxidation of $E$ FBCs which has been largely unexplored until to date due to the speculative instability of these isomers. The excellent control of reactivity for both $E-\mathbf{3}$ and $E-\mathbf{4}^{\prime}$ 'suggests that the methyl ester at C-18 stabilizes these highly reactive intermediates and their corresponding epoxide products by relieving electron density from the furan ring. More importantly, one can now fully appreciate the role of intertwined cyclase and oxidase phases in the conformational control of the 14-membered ring furanobutenolide cembranoids. The higher oxidation state for $E-3$, in lieu of a carbonyl group at C-14, enhanced the conformational strain imparted to the macrocycle backbone, likely through contributions of both Pitzer (torsional) and Prelog (transannular) strain. This extra strain forced the macrocycle into a more closed cup-like conformation thus blocking the C-19 methyl group to flip outside the macrocycle while exposing the $\Delta^{7,8}$ alkene $\alpha$-face for the stereoselective epoxidation. On the basis of the VT-NMR ${ }^{1} \mathrm{H}$ spectra, it can be established that the presence of a methylene at $\mathrm{C}-14$ relieves the macrocyclic strain leading to a dynamic equilibrium between two populations of conformers $E-\mathbf{4} / E-\mathbf{4}^{\prime}$ in a Curtin-Hammett situation (low transition-state energy barrier between conformers, $\left(\Delta G^{\ddagger}\right.$ of $\left.11.7 \mathrm{Kcal}_{\mathrm{mol}}{ }^{-1}\right)$ in which the most stable E-4' conformer $\left(\Delta G_{\text {rel }}=-0.303 \mathrm{Kcal}^{\prime} \mathrm{mol}^{-1}\right)$ can be stereoselectively epoxidized under kinetic control to deliver the $(7 R, 8 S)-9 b$. Again a peripheral attack from $\beta$-face of the computed lowest energy conformer $E-4^{\prime}$ explains well the opposite diastereochemical outcome of the epoxidation at $0{ }^{\circ} \mathrm{C}$. It can therefore be concluded that the level of oxidation at C-14 $(E-3$ vs $E-4)$ is a key conformational determinant that controls the macrocyclic strain in 14-membered ring $E$-FBCs. ${ }^{23,24}$

\section{Energy landscape of the dynamic equilibrium between populations of FBC-macrocycle conformers.}

To gain additional insights into the conformational landscape between the open (sandwich-like) and closed (cup-like) forms of FBC-macrocycles $E$-acerosolide (3) and $E$-deoxypukalide (4), we chose to probe the potential energy surfaces (PES) of these transformations by density functional theory (DFT) calculations (Figure 3). To determine potential activation barriers of the most favorable pathways, conformer structures inferred from NMR analysis (NOESY correlations and ${ }^{3} J_{\mathrm{HH}}$ scalar coupling constants, see Figure 2A) were exploited as starting points on the PES. Using relaxed coordinate scans, the macrocycles' conformational landscapes were obtained at the DFT B3LYP/3-21G level of theory through the incremental rotation of the furan-alkene $\pi$-system "in and out" of the macrocycle by pivoting around both $\mathrm{C}-7-\mathrm{C}-8-\mathrm{C}-9-\mathrm{C}-10$ and $\mathrm{C}-1-\mathrm{C}-2-\mathrm{C}-3-\mathrm{C}-4$ dihedral angles (37 increments each). The resulting energies calculated for $\sim 1,400$ conformations were used to generate a $2 \mathrm{D}-\mathrm{PES}$, which was examined to determine the impact of the $\Delta^{7,8}$ alkene internal rotation on the macrocycles' conformational space (see Supplementary Figures SI-8/9). Each potential energy minima on the PES was further optimized at the DFT B3LYP/6-31G(d) level of theory, and vibrational frequencies were calculated at the B3LYP/6$311 \mathrm{G}+(2 \mathrm{~d}, \mathrm{p})$ level using a conductor-like polarizable continuum model of chloroform (Figure 3). From these calculations, it was found that the global energy-minimum for acerosolide was a cup-like folded conformer $\mathbf{M I N}_{\mathbf{B}}$ in equilibrium with a much higher energy conformer $\mathbf{M I N}_{\mathbf{B}},(+5.8$ $\mathrm{kcal} / \mathrm{mol})$. Even if $\mathbf{M I N}_{\mathbf{B}}$, might represent a realistic intermediate, the activation barrier towards the transition state $\mathbf{T S}_{\mathbf{B}, \mathbf{B}}$, is so low $(0.9 \mathrm{Kcal} / \mathrm{mol})$ that $\mathbf{M I N} \mathbf{N}_{\mathbf{B}}$, is likely metastable, rapidly converting to $\mathbf{M I N}$. Furthermore, the central transition state $\mathbf{T S}_{\mathbf{A}^{\prime}, \mathbf{B}}$, governing the overall conformational interconversion between the macrocyclic open and closed forms was determined to be at $+13.2 \mathrm{Kcal} / \mathrm{mol}$ relative to the starting energy minima of $\mathbf{M I N}_{\mathbf{B}}$. While the transition state $\mathbf{T S}_{\mathbf{A}^{\prime}, \mathbf{B}}$ ' structure appears to sustain a large macrocyclic strain exemplified by a number of distorted bond angles, the perfectly aligned furan and alkene $\pi$-systems should contribute to its stabilization (Figure 3). In $\mathbf{T S}_{\mathbf{A}^{\prime}, \mathbf{B}}$ ', the butenolide and furan ring are also perfectly orthogonal. The geometric constraints imparted to the macrocyclic ring in the uphill path towards $\mathbf{T S}_{\mathbf{A}^{\prime}, \mathbf{B}}$, is associated with an allylic $\mathrm{A}^{1,3}$ strain between the Me19 group and the furan moiety which can be seen as a $s$-cis conformation of the C-5-C-6/C-7-C-8 diene system. Downhill from $\mathbf{T S}_{\mathbf{A}^{\prime}, \mathbf{B}}$ ' are two other local energy minima of open-form, $\mathbf{M I N}_{\mathbf{A}}$, and $\mathbf{M I N}_{\mathbf{A}}$, which are 2.1 and $1.1 \mathrm{Kcal} / \mathrm{mol}$ in energy above $\mathbf{M I N}_{\mathbf{B}}$, respectively. Considering the modest activation barrier $(c a .13 \mathrm{kcal} / \mathrm{mol})$ in the conformational pathway of acerosolide, the conformational preference towards $\mathbf{M I N}_{\mathbf{B}}$ can 
Figure 3. Computed macrocyclic conformational equilibrium pathway for acerosolide $E$-(3) and deoxypukalide $E$-(4) at $233 \mathrm{~K} .^{a}$

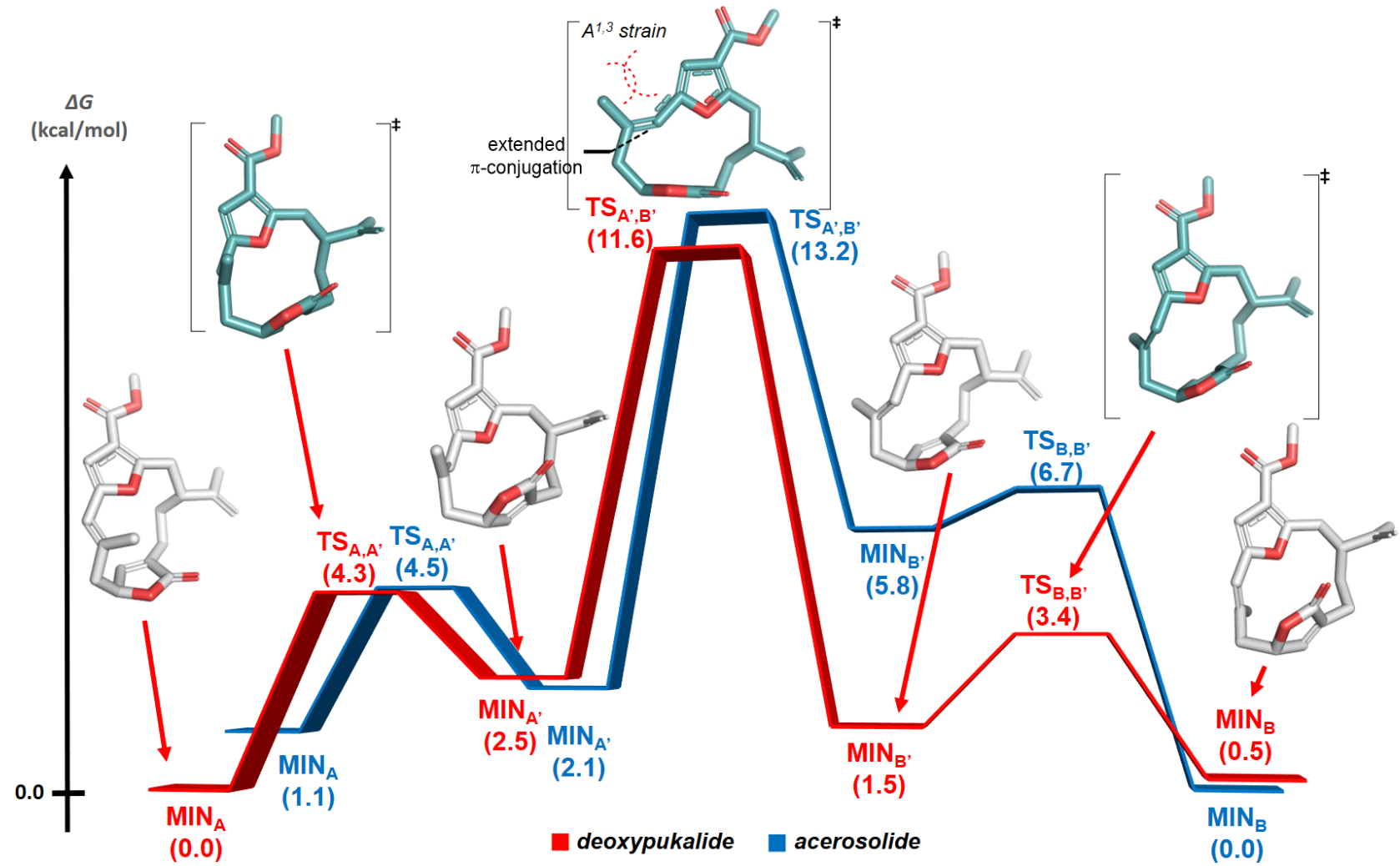

${ }^{a}$ Energy profile $(\Delta \mathrm{G}$ in $\mathrm{kcal} / \mathrm{mol})$ obtained at the DFT B3LYP/6-31G(d)//B3LYP/6-311+(2d,p) levels of theory for all optimized ground state

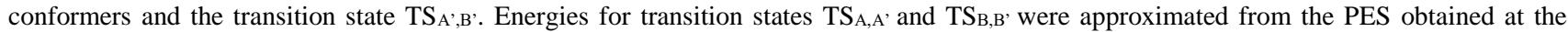
B3LYP/3-21G level of theory. Structures of acerosolide conformers omitted for simplicity, see Supporting Information Figure SI-12.

be explained by the Gibbs free energy difference $\Delta \Delta G\left(\mathbf{M I N}_{\mathbf{B}}\right.$ $\left.\mathbf{M I N}_{\mathbf{A}}\right)=-1.1 \mathrm{Kcal} / \mathrm{mol}$. In the lowest energy conformer of acerosolide $\mathbf{M I N}_{\mathbf{B}}$, the combination of an electrostatic interaction between the Me-19 and C-14 carbonyl, and a n- $\pi^{*}$ hyperconjation effect between the furan oxygen lone pair ( $p$ orbital) and the same C-14 carbonyl might afford stabilization to a more folded cup-like conformation (Figure Suplementary Figure SI-12). In contrast, in the PES of the deoxypukalide, a more open sandwich-like conformer $\mathbf{M I N}_{\mathbf{A}}$ was found as global minima of the macrocycle conformational space due to the absence of a carbonyl at C-14. The PES for the deoxypukalide conformational equilibrium highly resembles the path of acerosolide, with an ensemble of 4 stable energy minima (2 open- $\mathbf{M I N}_{\mathbf{A}} / \mathbf{M I N}_{\mathbf{A}}$, and 2 closed-conformers $\left.\mathbf{M I N}_{\mathbf{B}} / \mathbf{M I N}_{\mathbf{B}},\right)$. The pathway of interconversion for deoxypukalide presented a similar central transition state structure $\mathbf{T S}_{\mathbf{A}^{\prime}, \mathbf{B}},(+11.6 \mathrm{kcal} / \mathrm{mol})$ relative to the lowest energy conformation, $\mathbf{M I N}_{\mathbf{A}}$ in this case. Overall, the open-conformer, $\mathbf{M I N}_{\mathbf{A}}$, of deoxypukalide appears more stable with $\Delta G\left(\mathbf{M I N}_{\mathbf{A}}-\mathbf{M I N}_{\mathbf{B}}\right)=-0.5 \mathrm{Kcal} / \mathrm{mol}$. This computational result is in general agreement with the experimental equilibrium constant and ratios determined by NMR with $E-4$ ' being the major conformer as shown in Figure 2B. Furthermore, the DFT-estimated energy barrier $\left(\Delta G^{\ddagger}\right.$ of $11.6 \mathrm{Kcal} / \mathrm{mol}$ ) is consistent with the experimental interconversion barrier $E-\mathbf{4} / E-\mathbf{4}^{\prime}$ measured by the kinetic VTNMR experiments in $\mathrm{CDCl}_{3}$ at $233 \mathrm{~K}\left(\Delta G^{\ddagger}\right.$ of $\left.11.7 \mathrm{Kcal} / \mathrm{mol}\right)$. The conformational analysis for $E-\mathbf{3}$ and $E-\mathbf{4}$, and the transition state structure $\mathbf{T S}_{\mathbf{A}^{\prime}, \mathbf{B}}$, illustrated that the large isopropenyl side chain at $\mathrm{C}$-1 maintained a pseudo-equatorial position at all time during the $\Delta^{7,8}$ alkene rotation, ${ }^{23 \mathrm{~b}}$ while the small $\mathrm{H}-7$ hydrogen was the preferred group crossing the inside macrocyclic cavity to likely minimize transannular interactions (Supplementary Video-S1). Overall, the more strained $E-\mathbf{3}$ macrocycle is stabilized in a folded cup-like conformer with the butenolide ring blocking the $\Delta^{7,8}$ olefin $\beta$-face, while the more flexible $E-4$ macrocycle is stabilized in a sandwich-like conformation in which the butenolide ring rotated over thus hindering the opposite $\alpha$-face. This conformational analysis fully supports the reversal of stereoselectivity obtained experimentally from the epoxidation of $E-\mathbf{3}\left(\mathbf{M I N}_{\mathbf{B}}\right)$ and $E-\mathbf{4}\left(\mathbf{M I N}_{\mathbf{A}}\right)$ at low temperatures.

\section{Validation of the kinetic epoxidation strategy on $E$ - bipinnatin J methyl ether 10.}

To test this premise and expand the scope of the stereoselective epoxidation to a more realistic model towards bielschowskysin, the sequential photochemical isomerization and epoxidation have been evaluated on Z-bipinnatin $\mathbf{J}$ methyl ether $\mathbf{1 0}$ as outlined in Scheme 3. The systematic and foundational study from Pattenden on the $Z \rightarrow E$ photoisomerization of the $\Delta^{7,8}$ alkene revealed the labile nature of several $E$-FBCs $(e . g . \quad E-10) .{ }^{17}$ Seemingly, the $\pi-\pi^{*}$ photoexcitation of the $\Delta^{7,8}$ alkene moiety under UVB irradiation might trigger the formation of a 1,2-biradical intermediate which undergoes several competitive transformations, namely isomerization (desired in our case), ring contraction, and cyclopropanation. As expected, controlling the photoinduced $Z / E$-isomerization of bipinnatin $\mathrm{J}$ methyl ether Z-10 under UVB irradiation proved challenging, leading to the isolation of $E-\mathbf{1 0}$ in only $30 \%$ yield ( $87 \%$ brsm), along with the rearranged 12 - 
membered ring pseudopterane scaffold 11 (up to $12 \%$ yield). ${ }^{25,26}$ As previously suggested for the mechanism of the photocatalyzed Rodríguez-Pattenden ring contraction, a 1,2biradical intermediate might be initially generated to trigger a stereospecific suprafacial [1,3]-sigmatropic shift as the path to the ring-contracted product $\mathbf{1 1}$ with retention of the butenolide $(S)$-configuration. ${ }^{27}$ As shown in the sandwich-like conformation of $E-\mathbf{1 0}$, the C-9-C-10 $\sigma$-bond migration likely arise from the $\alpha$-face at C-8 via a concerted suprafacial $[1,3]$ sigmatropic shift -authorized by the orbital symmetry rules for $\left[\sigma 2_{\mathrm{s}}+\pi 2_{\mathrm{s}}\right]$ cycloaddition reaction- to obtain the $(7 S)$ configuration observed in $\mathbf{1 1}^{28}$ No evidence of other diastereomers could be found on the ${ }^{1} \mathrm{H}$ NMR spectra of 11 . Never the less, by keeping irradiation times shorter than 10 minutes, the photoisomer $E$-10 was intercepted and isolated in a pure form. The stereoselective epoxidation of $E-\mathbf{1 0}$ with DMDO at low temperatures $\left(-40\right.$ to $\left.-20{ }^{\circ} \mathrm{C}\right)$ provided $(7 R, 8 S)$ epoxide 12 in $30 \%$ yield. The observed stereoselectivity was again in general agreement with the DFT caluculations for $E$ 10 in which the open sandwich-conformer, $\mathbf{M I N}_{\mathbf{A}}$, appeared more stable with a $\Delta G\left(\mathbf{M I N}_{\mathbf{A}}-\mathbf{M I N}_{\mathbf{B}}\right)=-1.6 \mathrm{Kcal} / \mathrm{mol}$ (see Supplementary Table SI-4). In agreement with our stereochemical model, the ${ }^{1} \mathrm{H}$ NMR analysis of the reaction mixture revealed no measurable concentration of other diastereomers. Epoxide $\mathbf{1 2}$ is the most unstable epoxide from the entire synthetic series (i.e. 8a-d). Upon longer reaction time of epoxidation (18 hours) or standing in a freezer, 12 undergoes a gradual dearomative hydrolysis to the ene-dione product 13 . Given the instabilities of both $E-\mathbf{1 0}$ and 12, and the restricted amounts of bipinnatin $\mathrm{J}$ available to us, this route was not further pursued.

Scheme 2. Photoisomerization of bipinnatin J methyl ether Z-10 into $E-10$ and the ensuing stereocontrolled epoxidation under kinetic control.

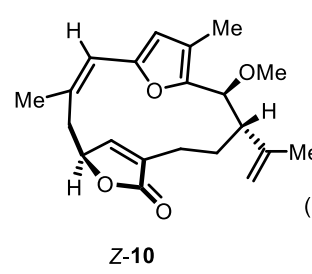

$Z-10$

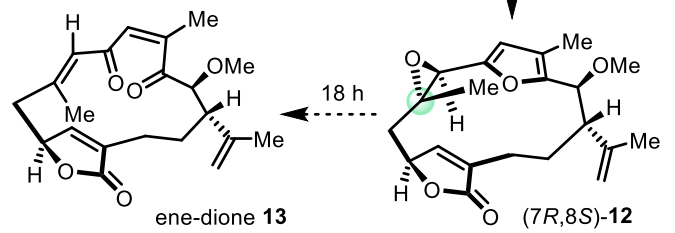

$E-10$

2. DMDO $30 \%$ yield

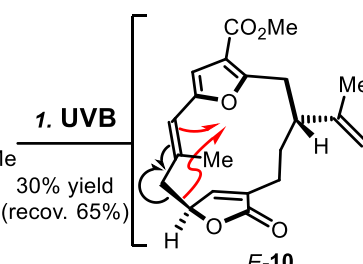

${ }^{a}$ Reagents and conditions: (1) UVB $(\lambda=300 \mathrm{~nm})$, Pyrex filter, $\mathrm{CD}_{3} \mathrm{CN}, \mathrm{rt},<10 \mathrm{~min}$. (2) DMDO (1.2 eq.), $\mathrm{CH}_{2} \mathrm{Cl}_{2}(0.2 \mathrm{M}),-40$ to $-20{ }^{\circ} \mathrm{C}, 1.5 \mathrm{~h}$.

Taken together, the results on the photoisomerization and stereoselective epoxidation from several representative members of the 14-membered ring class of FBC-macrocycles $(E-3, Z-3, E-4, E-10$ and $Z-10)$ support several biosynthetic hypotheses and inform about the key structural determinant for the C-7-C-8 stereocontrol. As previously eluded, the degree of oxidation at $\mathrm{C}-18$ is crucial in tuning the furan reactivity (initiating dearomatization), and avoiding over-oxidation. Furthermore, another lesson learned from the oxidase phase revealed that the $s p^{2}$ C-14 carbonyl moiety found in acerosolide $E-3$ alter the macrocycle conformational equilibrium as such that one major conformer population can be observed, leading to a highly $\alpha$-stereoselective epoxidation of the $\Delta^{7,8}$ alkene. In contrast, the switch of conformational preference in $E-4$ and $E$ 10 suggests that a lower oxidation state (methylene at C-14) released macrocyclic strain which in turn enables a stereoselective epoxidation to take place from the $\beta$-face under kinetic control $\left(-40\right.$ to $\left.0{ }^{\circ} \mathrm{C}\right)$. This $\alpha$ : $\beta$ selectivity control in the epoxidation of $E$-FBCs strongly suggests that $Z$-metabolites represent the in vivo reservoir of metabolites to achieve regioselective oxidations (certainly based on several factors such as each individual coral genome, organism yearly reproduction cycle and ecological niche) that intertwine both cyclase and oxidase phases to deliver specific metabolites on demand.

\section{Kinetic versus thermodynamic control of the dearomatization of furanocembranoids.}

Fenical was first to report a FBC-macrocycle artifact of isolation derived from lophotoxin bearing a dearomatized furan as an exo-enol ether moiety. ${ }^{29}$ Since then, other exo-enol ethers have been genuinely isolated, ${ }^{30}$ leading to the hypothesis that exo-enol ethers (e.g. 15) might be plausible biosynthetic precursors to various transannular reactions. ${ }^{2}$ The seemingly straightforward conversion of the furanyl epoxide moiety present in macrocycles $\mathbf{8}$ into the corresponding dearomatized exo-enol ethers 15 revealed to be the most challenging step of the synthesis. Earlier studies by Rodríguez ${ }^{11 a}$, Pattenden ${ }^{12 a}$, Mulzer ${ }^{12 b}$, and Trauner ${ }^{12 c}$ established the difficulties to devise a chemo-, regio- and stereoselective dearomatization strategy which informed our own orthogonal approach of the dearomatization under kinetic control. ${ }^{10}$ To this aim, trans epoxides $\mathbf{8 a}, \mathbf{b}$ and cis epoxide 8c were obtained from the direct epoxidations of the corresponding $E-\mathbf{3}$ and Z-3 macrocycles respectively (see Scheme 1). The cis $(7 S, 8 S)$-epoxide 8d was prepared in $56 \%$ yield over 2 steps from $\mathbf{8 b}$ by successive treatments with TMSCl, and AgF in benzene (Scheme 3). Having in hand the four cis/trans diastereomer epoxides of acerosolide 8a-d, our initial dearomatization study could be completed to obtain a detailed stereochemical blueprint of the kinetic control. As shown on Scheme 3, epoxides 8a-d endured a nucleophilic opening in a highly regio- and stereoselective manner to generate the corresponding C-7-C-8 chlorohydrins 14a-d with a suprisingly impeccable retention of configuration at $\mathrm{C}-7 .{ }^{31}$ Despite their inherent instability, these chlorohydrins were carefully isolated and characterized to secure their relative stereochemistry. ${ }^{10}$ These results were rationalized by a peripheral kinetic attack of the chloride nucleophile from the less hindered $\alpha$ - or $\beta$-face of the vinylogous oxocarbeniums $\mathbf{A}$ or $\mathbf{B}$ respectively (Scheme 3). ${ }^{32}$ Likewise, the regioselective $\mathrm{C}-3$ dearomatization of these chlorohydrins was successfully achieved with AgOTf and $\mathrm{MeOH}$ to deliver the corresponding $(Z)$-exo enol ethers 14a-d in $23-36 \%$ yields with an exquisite transfer of chirality $(\geq 20: 1$ $\mathrm{dr} ; \geq 4: 1 \mathrm{rr}$ ). This precise substrate-control in each epoxide opening and dearomatization reactions under kinetic conditions suggests that both steps proceeded through the same $S_{N} 1$-type 
Scheme 3. One-pot synthesis of (Z)-exo-enol ethers 15a-c through successive highly regio- and stereoselective kinetic dearomatizations.
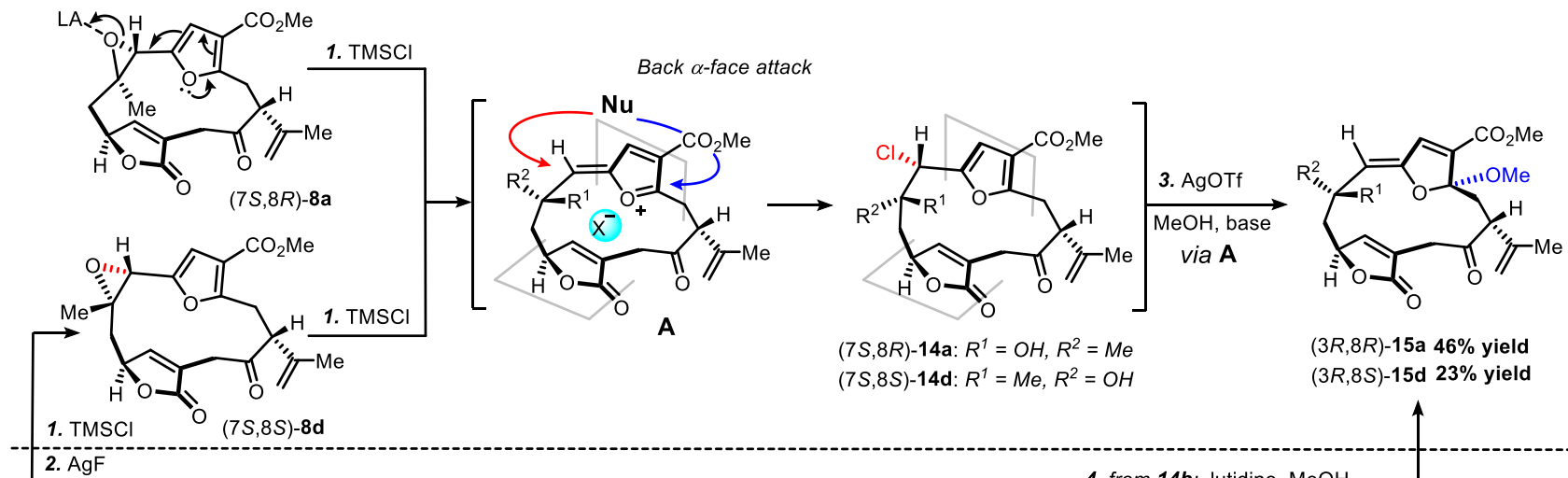

A

(7S,8R)-14a: $R^{1}=\mathrm{OH}, R^{2}=M e$

$(7 S, 8 S)-14 \mathrm{~d}: R^{1}=\mathrm{Me}, R^{2}=\mathrm{OH}$

$(3 R, 8 R)-15$ a $46 \%$ yield $(3 R, 8 S)-15 d \mathbf{d} 23 \%$ yield

$$
\text { 2. AgF }
$$

$56 \%$ yield

yield
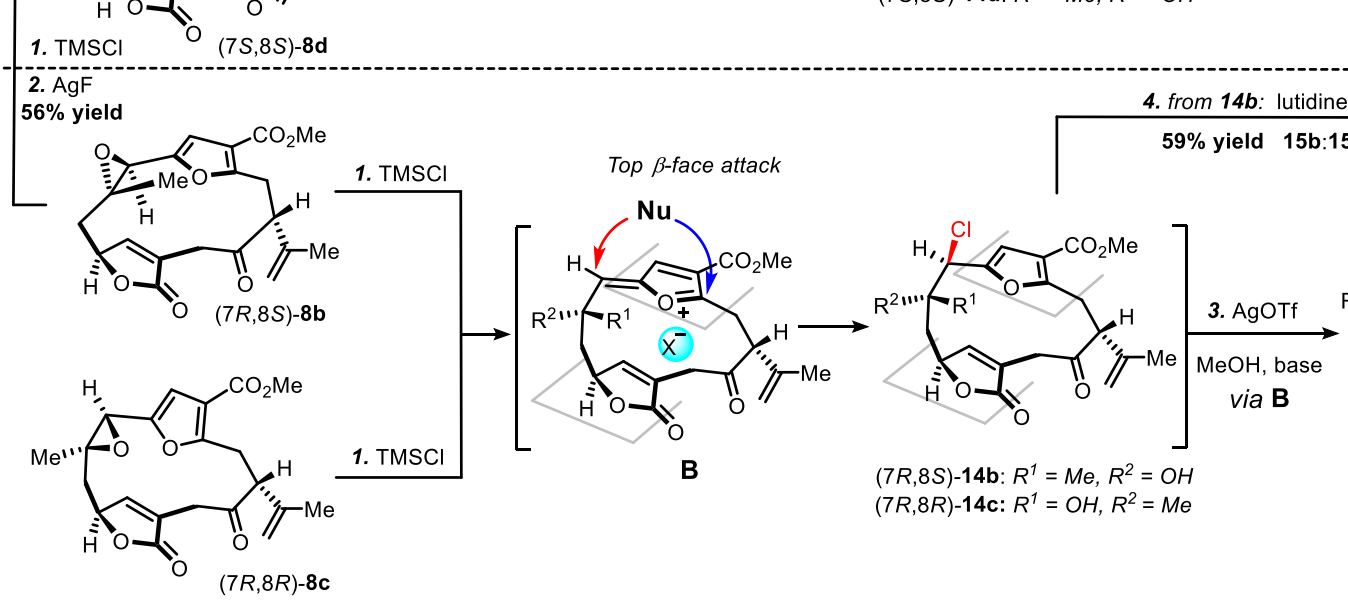

(7R,8S)-14b: $R^{1}=M e, R^{2}=\mathrm{OH}$ $(7 R, 8 R)-14 \mathrm{c}: R^{1}=\mathrm{OH}, R^{2}=\mathrm{Me}$

${ }^{a}$ Reagents and conditions: (1) TMSCl (1.1 eq.), $0{ }^{\circ} \mathrm{C}, \mathrm{CH}_{2} \mathrm{Cl}_{2}$ (20-50 mM), 1-2 h. (2) AgF (1.0 eq.) benzene, rt, 0.5 h. (3) AgOTf (1.1-1.5 eq.), $78{ }^{\circ} \mathrm{C}, \mathrm{CH}_{2} \mathrm{Cl}_{2}$ (25-50 mM), $\mathrm{MeOH}$ (5.0-7.0 eq.), lutidine (1.1-1.4 eq.), 3 h. (4) lutidine (4.6 eq.), MeOH (258 eq.), rt, 12 h.

scenario. Indeed, the (7S)-epoxides $\mathbf{8 a} / \mathbf{8 d}$ opening as well as the ensuing (7S)-chlorohydrins $\mathbf{1 4 a} / \mathbf{1 4 d}$ dearomatizations are proposed to proceed via oxocarbenium $\mathbf{A}$ in which the orthogonality between the butenolide and furanoxonium rings (cup-like conformation) supports a nucleophilic attack at the $\mathrm{C}$ 7 or C-3 positions from the convex $\alpha$-face. In a similar $\mathrm{S}_{\mathrm{N}} 1$-type scenario, (7R)-epoxides $\mathbf{8 b} / \mathbf{8 c}$ opening and (7R)-chlorohydrins $\mathbf{1 4 b} / \mathbf{1 4 c}$ dearomatizations likely proceeded via oxocarbenium $\mathbf{B}$ in which the butenolide and furanoxonium rings are more in a parallel (sandwich-like) arrangement, thus blocking the concave face and opening up the top $\beta$-face for nucleophilic attack. Collectively these results suggest that under kinetic control, the initial epoxides' configurations at C-7 can be exquisitely transferred into the C-3 configurations of the $(Z)$ exo enol ether ketal products (retention relative of configuration). Having completed a cohesive model of kinetic stereoselectivity based upon the conformational bias of oxocarbeniums $\mathbf{A} / \mathbf{B}$, the dearomatization of chlorohydrin $\mathbf{1 4 b}$ was attempted under a set of thermodynamic conditions. Treating 14b with lutidine and $\mathrm{MeOH}$ at room temperature mostly avoided the issue of rapid rearomatization previously reported by Pattenden $(2: 1 \mathrm{rr}),{ }^{12 \mathrm{a}}$ therefore leading to an efficient dearomatization $(59 \%$ yield $)$ despite the diastereomeric mixture of products $\mathbf{1 5 b} / \mathbf{1 5 d}(7: 1 \mathrm{dr})$. The erosion of diastereoselectivity observed in this first successful example of thermodynamic dearomatization suggested that a conformational equilibrium between oxocarbeniums $\mathbf{A}$ and $\mathbf{B}$ might take place at ambient temperature.

With these results in hand, a biomimetic endgame was attempted from both cis $(7 S, 8 S)$-epoxides $\mathbf{8 d}$ and $9 d$ respectively derived from acerosolide and deoxypukalide (Scheme 4A). Recall that 9d was synthesized in 3 steps ca. $35 \%$ overall yield with high stereocontrol (Scheme 1). Epoxide opening was achieved under the kinetic conditions described above, leading to isolate the corresponding chlorohydrins. ${ }^{18}$ After simple evaporation of volatiles, the chlorohydrin intermediates were dearomatized in the same pot under thermodynamic control by the addition of lutidine and $\mathrm{MeOH}$ to afford both (Z)-exo enol ether ketal products $\mathbf{1 5 d} / \mathbf{1 6 d}$. These (Z)-exo enol ethers were then photoisomerized under UVC irradiation to achieve a transannular [2+2] photocycloaddition into the bielschowskyane photoproducts $18 d$ and 19d in $19 \%$ and $8 \%$ overall yields over 3 steps. ${ }^{15 \mathrm{c}-\mathrm{d}, 16,33}$ These one-pot transformations are modestly efficient, yet complete our initial study on bielschowskyane analogs by controlling for the first time the desired $(8 S)$-configuration. As initially suggested by Nicolaou $^{16}$ from an in situ ${ }^{1} \mathrm{H}$ NMR monitoring of the photoreaction, the photoisomerized $(E)$-exo enol ether (e.g. 17a) appeared to be the reactive intermediate in the $[2+2]$ photocycloaddition. The isolation of $\mathbf{1 7 a}$ allowed us to verify that neither $(Z)$ - nor $(E)$-exo enol ethers underwent a thermal cycloaddition. As shown in Figure 4, the strong long-range NOESY correlation observed only in the $(E)$-exo enol ether 17a (not in 15a) between the $\mathrm{H}-7$ and $\mathrm{H}-11$ protons suggested that the two olefins C-7-C-8 and C-11-C-12 were in close spatial proximity, as one would expect for the $\pi$-systems to properly align for the [2+2] photocycloaddition..$^{33,34}$ Macrocycle 17a was further irradiated with UVC to successfully deliver 18a, which confirms Nicolaou's hypothesis. Having access to the novel $(7 S, 8 S)$-epoxides $\mathbf{8 d}$ and $9 d$ enabled us to complete the syntheses of the complex fuse-bridged tricyclo[9.3.0.0]tetradecane skeleton of bielschowskysin with the appropriate 8 stereocenters (6 of which are contiguous). These results help rationalize the link between the proposed 
Scheme 4. A. Endgame towards bielschowskyane analogs (8S)-18d and 19d under thermodynamic control. B. Cascade reaction for the direct epoxide transformation under photoirradiation to the bielschowskyane C8-epimer (8R)-18a.

A
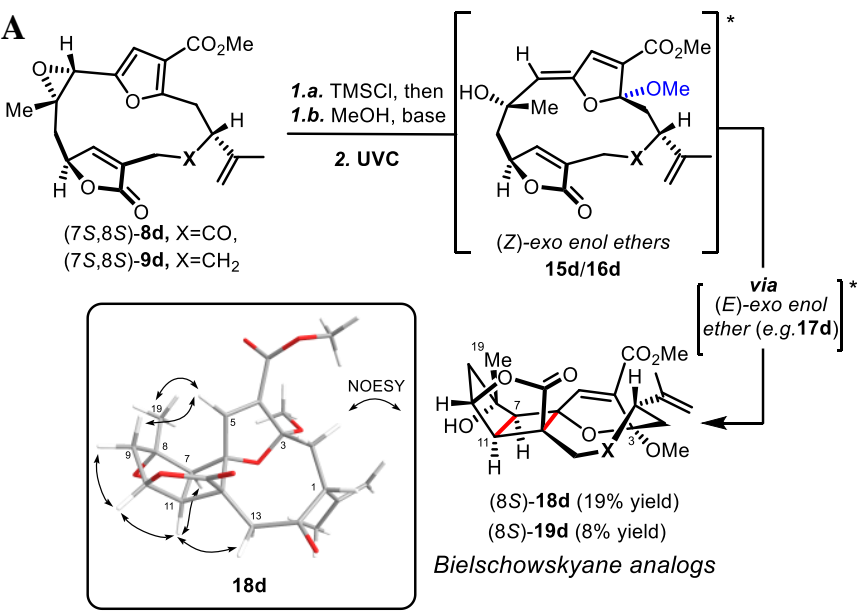

$\mathbf{B}$
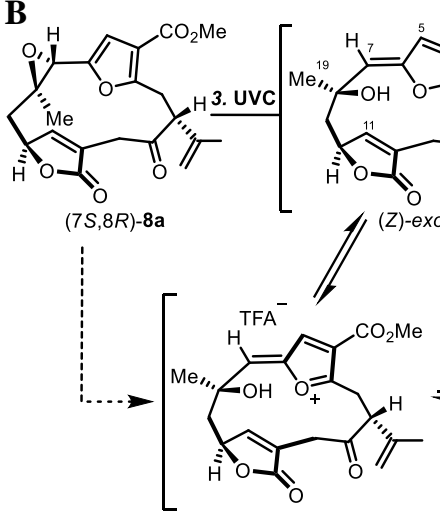

(20)

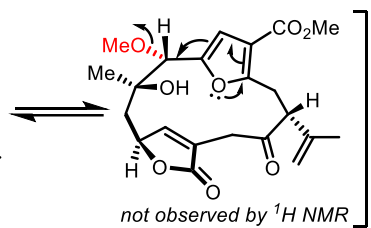

${ }^{a}$ Reagents and conditions: (1) a. TMSCl (1.1 eq.), $0{ }^{\circ} \mathrm{C}, \mathrm{CH}_{2} \mathrm{Cl}_{2}, 1 \mathrm{~h}$, then evaporation of volatiles and $\mathrm{b}$. $\mathrm{MeOH}$ (excess), lutidine (4.6 or 4.3 eq.), $\mathrm{CH}_{2} \mathrm{Cl}_{2}, 12$ h, rt. (2) UVC ( $\lambda=254 \mathrm{~nm}$ ), $\mathrm{CDCl}_{3}, \mathrm{rt}, 2$ h. (3) UVC $(\lambda=254 \mathrm{~nm}), \mathrm{MeOH}$ (48 eq.), TFA (2.5 eq. $), \mathrm{CDCl}_{3}(26 \mathrm{mM}), 1 \mathrm{~h}$.

biosynthetic steps towards bielschowskysin and expose the role played by the macrocyclic conformational preferences in controlling an exquisite stereoinduction at $\mathrm{C}-7$, and the retentive stereotransfer to $\mathrm{C}-3$ during dearomatization. Given the initial success of dearomatization under thermodynamic control, a dearomative cascade reaction was also tailored in presence of methanol and trifluoroacetic acid acting as Brønsted acid (and potentially a weak nucleophile). Excitingly, under UVC-photoirradiation both dearomatization and [2+2] photocycloaddition took place rapidly in one-pot to produce the 8-epi-bielschowskyane analog $\mathbf{1 8 a}$ in $46 \%$ overall yield. ${ }^{35} \mathrm{H}$

Figure 4. Isolation and conformational characterization of the photo-isomerized intermediate $(E)$-exo enol ether 17a

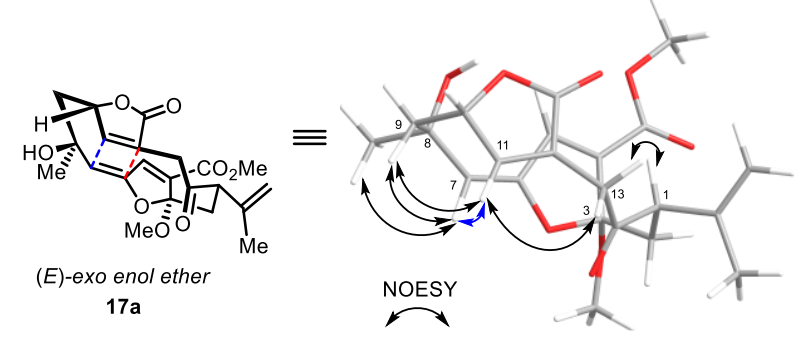

NMR Monitoring of the cascade reaction revealed no measurable concentration of the rearomatized C-7 methyl ether regioisomer as expected from an acid-mediated reaction, suggesting that under these mild acidic conditions, dearomatization via 15a can still take place to feed the [2+2] photocycloaddition in a cascade manner.

Conclusion. E-FBC-macrocycles have been rarely isolated due to their doubtful instability in comparison to their Z-FBC analogs. The study herein confirms this hypothesis, but also demonstrates that the additional strain imparted by the $\Delta^{7,8}$ alkene of $E$-configuration controls the accessible macrocyclic conformational space to direct reactivity. Our comparative study on $E$-acerosolide (3), $E$-deoxypukalide (4), and bipinnatin $\mathrm{J}$ methyl ether (10) shed new light on the original work from Pattenden as we now showed that both $E / Z$ and reverse $Z / E$ photoisomerization of the $\Delta^{7,8}$ olefin can be achieved within these macrocycles. We have shown that the conformational strain associated with the C-14 carbonyl moiety of $E$ acerosolide forces the macrocycle into a folded cup-like conformer which completely blocks several photochemical pathways typically observed in more flexible FBC-macrocycles of lower oxidation state $\mathbf{1 , 2}$ or $\mathbf{4}$ to occur (e.g. cyclopropanation to gersolanes, and ring contraction to pseudopteranes). ${ }^{27,28}$ Results from NMR NOESY experiments and DFT calculations further supported that $E-\mathbf{3}$ and $E-\mathbf{4}$ populated different conformational lowest-energy minima $\left(\Delta \Delta G\left(\mathbf{M I N}_{\mathbf{A}}-\mathbf{M I N}_{\mathbf{B}}\right)=\right.$ -0.5 and $+1.1 \mathrm{Kcal} / \mathrm{mol}$ respectively) which are in full agreement with the switch of facial selectivity observed in epoxidations under kinetic control. Indeed, the high $\beta$ selectivity obtained for the first time in the epoxidations of the more flexible $E-\mathbf{4}$ and $E-\mathbf{1 0}$ macrocycles was rationalized by a conformational dynamic equilibrium operating under a CurtinHammett scenario in which the more stable sandwich-like conformers have their $\Delta^{7,8}$ olefin $\alpha$-face shielded by the remote butenolide ring (as opposed to the cup-like conformer of $E-3$ ). By taking advantage of the macrocyclic conformational bias at low temperature, a synthetic sequence was established for the pivotal regio- and stereoselective furan dearomatization triggered by epoxide ring-opening. The facial discrimination in the substitutions at C-7 forming the chlorohydrin intermediates and the successive dearomatizations at C-3 occurred with exquisite retention of configuration ( $\mathrm{S}_{\mathrm{N}} 1$-like mechanism). This kinetic phenomenon is once again likely imparted by the innate conformational bias expected in the macrocyclic vinylogous oxocarbenium intermediates as confirmed by the erosion of diastereoselectivity observed under thermodynamic conditions. This novel conformational knowledge ${ }^{36}$ applied to $E-\mathbf{4}$ resulted in a 6-step synthesis of the intricate tricyclo[9.3.0.0]tetradecane bielschowskysin mimic 19d (3\% overall yield) harboring 8 of the 11 stereogenic centers present in the natural product. Even so, a thermodynamic cascade reaction mediated by Brønsted acid under photoirradiation was devised with the direct epoxide opening and transannular [2+2] photocycloaddition to synthesize another bielschowskyane analog in a single step (8a $\rightarrow$ 18a). Collectively, our results demonstrated for the first time that the $E$-configured $\Delta^{7,8}$ alkene moiety is crucial in controlling the relative and absolute stereogenicity of the downstream steps towards the $(3 S, 7 S, 8 S)$-bielschowskysin.

Collectively, our results establish that functional groups at specific positions on the FBC-skeleton foster conformational steering of the macrocycles, which provides a new model for predicting kinetic reactivity and stereoselectivity towards 
several other polycyclic FBC natural products 5-6. The syntheses described herein highlight the importance of conformational analysis in macrocycles to fully exploit the inherent reactivity of these strained molecules and uncover potential synthetic strategies. ${ }^{37}$ As highlighted by Stoltz in a recent review article, only a single de novo synthesis of polycyclic FBC natural products has been reported since the $1990 \mathrm{~s}^{2 \mathrm{c}}$ Given the remaining synthetic challenges associated with this family of natural products (e.g. verrilin, plumarellide, or mandapamate), we anticipate that both strategies of macrocyclic $E$-alkene epoxidation and kinetic/thermodynamic dearomatization reported herein will find applications to the synthesis of a large number of molecules in this class.

\section{ASSOCIATED CONTENT}

\section{Supporting Information}

The Supporting Information is available free of charge on the ACS Publications website.

Tables of selected results from dearomatization optimization under kinetic and thermodynamic control are reported. Isolation and synthetic procedures, DFT calculations, VT-NMR study and characterization data including ${ }^{1} \mathrm{H},{ }^{13} \mathrm{C}$ and NOESY spectra are available online.

\section{AUTHOR INFORMATION}

\section{Corresponding Author}

*Correspondence should be addressed to S.P.R. Email: sroche2@fau.edu or L.M.W Email: $\underline{\text { lwest@ fau.edu }}$

\section{Author Contributions}

This project was conceived by S.P.R. and L.M.W.; P.D.S. performed the metabolites isolation and research experiments, and S.P.R. with L.M.W. and P.D.S. analyzed the data. The manuscript was written by S.P.R.; All authors have given approval to the final version of the manuscript.

Keywords: natural products $\bullet$ cembrane - furanocembranoid • bielschowskysin $\bullet$ kinetic dearomatization.

\section{ACKNOWLEDGMENT}

We would like to thank Dr. K. B. Basso at the Mass Spectrometry Research and Education Center from the Department of Chemistry at the University of Florida for the high-resolution mass spectrometry analysis supported by the NIH (S10 OD021758-01A1).

\section{REFERENCES}

(1) Berrue, F.; Kerr, R. G. Diterpenes from gorgonian corals. Nat. Prod. Rep. 2009, 26, 681-710.

(2) For an extensive overview of FBC chemistry, see the following excellent reviews: (a) Roethle, P. A.; Trauner, D. The chemistry of marine furanocembranoids, pseudopteranes, gersolanes, and related natural products. Nat. Prod. Rep. 2008, 25, 298-317. (b) Li, Y.; Pattenden, G. Perspectives on the structural and biosynthetic interrelationships between oxygenated furanocembranoids and their polycyclic congeners found in corals. Nat. Prod. Rep. 2011, 28, 1269-1310. (c) Craig, R. A.; Stoltz, B. M. Polycyclic Furanobutenolide-Derived Cembranoid and Norcembranoid Natural Products: Biosynthetic Connections and Synthetic Efforts. Chem. Rev. 2017, 117, 7878-7909. (d) Palframan, M. J.; Pattenden, G. Biosynthetic Interrelationships within Polycyclic Cembranoids Isolated from Corals: Conjecture, Biomimetic Synthesis and Reality. Eur. J. Org. Chem. 2020, 2330-2349.
(3) Croteau, R.; Ketchum, R. E. B.; Long, R. M.; Kaspera, R.; Wildung, M. R. Taxol Biosynthesis and Molecular Genetics. Phytochem. Rev. 2006, 5, 75-97.

(4) (a) Chen, K.; Ishihara, Y.; Galán, M. M.; Baran, P. S. Total synthesis of eudesmane terpenes: cyclase phase. Tetrahedron 2010, 66, 4738-4744. (b) Foo, K.; Usui, I.; Goetz, D. C. G.; Werner, E. W.; Holte, D.; Baran, P. S. Scalable, Enantioselective Synthesis of Germacrenes and Related Sesquiterpenes Inspired by Terpene Cyclase Phase Logic. Angew. Chem. Int. Ed. 2012, 51, 11491-11495.

(5) (a) Chen, K.; Baran, P. S., Total synthesis of eudesmane terpenes by site-selective C-H oxidations. Nature 2009, 459, 824-828. (b) McKerrall, S. J.; Joergensen, L.; Kuttruff, C. A.; Ungeheuer, F.; Baran, P. S., Development of a Concise Synthesis of (-)-Ingenol. J. Am. Chem. Soc. 2014, 136, 5799-5810. (c) Kanda, Y.; Nakamura, H.; Umemiya, S.; Puthukanoori, R. K.; Murthy Appala, V. R.; Gaddamanugu, G. K.; Paraselli, B. R.; Baran, P. S. Two-Phase Synthesis of Taxol. J. Am. Chem. Soc. 2020, 142, 10526-10533.

(6) (a) Itoh, T.; Tokunaga, K.; Matsuda, Y.; Fujii, I.; Abe, I.; Ebizuka, Y.; Kushiro, T. Reconstitution of a Fungal Meroterpenoid Biosynthesis Reveals the Involvement of a Novel Family of Terpene Cyclases. Nature Chem. 2010, 2, 858-864. (b) Mitsuhashi, T.; Barra, L.; Powers, Z.; Kojasoy, V.; Cheng, A.; Yang, F.; Taniguchi, Y.; Kikuchi, T.; Fujita, M.; Tantillo, D. J.; Porco Jr, J. A.; Abe, I. Exploiting the Potential of Meroterpenoid Cyclases to Expand the Chemical Space of Fungal Meroterpenoids. Angew. Chem. Int. Ed. 2020, 59 ahead of print https://doi.org/10.1002/anie.202011171

(7) (a) Baunach, M.; Franke, J.; Hertweck, C. Terpenoid Biosynthesis Off the Beaten Track: Unconventional Cyclases and Their Impact on Biomimetic Synthesis. Angew. Chem. Int. Ed. 2015, 54, 2604-2626. (b) Zhang, G.-Q.; Xu, Q.; Bian, C.; Tsai, W.-C.; Yeh, C.-M.; Liu, K.W.; Yoshida, K.; Zhang, L.-S.; Chang, S.-B.; Chen, F.; Shi, Y.; Su, Y.Y.; Zhang, Y.-Q.; Chen, L.-J.; Yin, Y.; Lin, M.; Huang, H.; Deng, H.; Wang, Z.-W.; Zhu, S.-L.; Zhao, X.; Deng, C.; Niu, S.-C.; Huang, J.; Wang, M.; Liu, G.-H.; Yang, H.-J.; Xiao, X.-J.; Hsiao, Y.-Y.; Wu, W. L.; Chen, Y.-Y.; Mitsuda, N.; Ohme-Takagi, M.; Luo, Y.-B.; Van de Peer, Y.; Liu, Z.-J. The Dendrobium catenatum Lindl. genome sequence provides insights into polysaccharide synthase, floral development and adaptive evolution. Sci. Rep. 2016, 6, 19029. (c) Rudolf, J. D.; Chang, C.-Y. Terpene Synthases in Disguise: Enzymology, Structure, and Opportunities of Non-Canonical Terpene Synthases. Nat. Prod. Rep. 2020, 37, 425-463.

(8) Reyes, E.; Uria, U.; Carrillo, L.; Vicario, J. L., Transannular reactions in asymmetric total synthesis. Tetrahedron 2014, 70, 94619484.

(9) (a) Kimbrough, T. J. Synthesis of Coralloidolide B, Coralloidolide C, a Photochemical Synthesis of Intricarene, and Progress Towards the Synthesis of Bielschowskysin. Ph.D. Dissertation, University of California, Berkeley, CA, 2011. (b) Toelle, N.; Weinstabl, H.; Gaich, T.; Mulzer, J. Light-Mediated Total Synthesis of 17-Deoxyprovidencin. Angew. Chem. Int. Ed. 2014, 53, 3859-3862.

(10) Scesa, P.; Wangpaichitr, M.; Savaraj, N.; West, L.; Roche, S. P. A Kinetic Dearomatization Strategy for an Expedient Biomimetic Route to the Bielschowskysin Skeleton. Angew. Chem. Int. Ed. 2018, 57, 1316-1321.

(11) (a) Marrero, J.; Benítez, J.; Rodríguez, A. D.; Zhao, H.; Raptis, R. G., Bipinnatins K-Q, Minor Cembrane-Type Diterpenes from the West Indian Gorgonian Pseudopterogorgia kallos: Isolation, Structure Assignment, and Evaluation of Biological Activities. J. Nat. Prod. 2008, 71, 381-389. (b) McAulay, K.; Clark, J. S., Total Synthesis of $7-$ epi-Pukalide and 7-Acetylsinumaximol B. Chem. Eur. J. 2017, 23, 9761-9765.

(12) For earlier studies highlighting some of the challenges associated with the biomimetic furan dearomatization strategy on FBCmacrocycles, see: (a) Li, Y.; Pattenden, G.; Rogers, J. Synthesis of exo enol ether-cyclic ketal isomers of substituted furanmethanol structures related to marine furanocembranoids. Tetrahedron Lett. 2010, 51, 1280-1283. (b) Farcet, J.-B.; Himmelbauer, M.; Mulzer, J. Photochem- 
ical and Thermal $[2+2]$ Cycloaddition to Generate the Bicyclo[3.2.0]heptane Core of Bielschowskysin. Eur. J. Org. Chem. 2013 4379-4398. (c) Stichnoth, D.; Kölle, P.; Kimbrough, T. J.; Riedle, E.; de Vivie-Riedle, R.; Trauner, D. Photochemical formation of intricarene. Nat. Commun. 2014, 5, 5597.

(13) Dorta, E.; Diaz-Marrero, A. R.; Brito, I.; Cueto, M.; D'Croz, L.; Darias, J., The oxidation profile at $\mathrm{C}-18$ of furanocembranolides may provide a taxonomical marker for several genera of octocorals. Tetrahedron 2007, 63, 9057-9062.

(14) Li, Q.; Qian, H.; Shao, B.; Hughes, R. P.; Aprahamian, I. Building Strain with Large Macrocycles and Using It To Tune the Thermal Half-Lives of Hydrazone Photochromes. J. Am. Chem. Soc. 2018, 140, 11829-11835.

(15) (a) Miao, R.; Gramani, S. G.; Lear, M. J. Stereocontrolled entry to the tricyclo[3.3.0]oxoheptane core of bielschowskysin by a [2+2] cycloaddition of an allene-butenolide. Tetrahedron Lett. 2009, 50, 1731-1733. (b) Farcet, J.-B.; Himmelbauer, M.; Mulzer, J. A Non-Photochemical Approach to the Bicyclo[3.2.0]heptane Core of Bielschowskysin. Org. Lett. 2012, 14, 2195-2197. (c) Jana, A.; Mondal, S.; Firoj Hossain, M.; Ghosh, S. Stereocontrolled approach to the highly functionalized bicyclo[3.2.0] heptane core of bielschowskysin through intramolecular $\mathrm{Cu}(\mathrm{I})$-catalyzed [2+2] photocycloaddition. Tetrahedron Lett. 2012, 53, 6830-6833. (d) Townsend, S. D.; Sulikowski, G. A. Progress toward the Total Synthesis of Bielschowskysin. Org. Lett. 2013, 15, 5096-5098. (e) Himmelbauer, M.; Farcet, J.-B.; Gagnepain, J.; Mulzer, J. A Palladium-Catalyzed Carbo-oxygenation: The Bielschowskysin Case. Org. Lett. 2013, 15, 3098-3101. (f) Himmelbauer, M.; Farcet, J.-B.; Gagnepain, J.; Mulzer, J. An Approach to the Carbon Backbone of Bielschowskysin. Part 1: Photocyclization Strategy. Eur. J. Org. Chem. 2013, 2013, 8214-8244. (g) Farcet, J.-B.; Himmelbauer, M.; Mulzer, J. An Approach to the Carbon Backbone of Bielschowskysin, Part 2: Non-Photochemical Strategy. Eur. J. Org. Chem. 2013, 2013, 8245-8252. (h) Meyer, M. E.; Phillips, J. H.; Ferreira, E. M.; Stoltz, B. M. Use of a palladium(II)-catalyzed oxidative kinetic resolution in synthetic efforts toward bielschowskysin. Tetrahedron 2013, 69, 7627-7635. (i) Yang, E. G.; Sekar, K.; Lear, M. J. A macrolactonisation approach to the cembrane carbocycle of bielschowskysin. Tetrahedron Lett. 2013, 54, 4406-4408. (j) Jana, A.; Mondal, S.; Ghosh, S. Studies towards the synthesis of bielschowskysin. Construction of the highly functionalized bicyclo[3.2.0]heptane segment. Org. Biomol. Chem. 2015, 13, 1846-1859. (k) Farcet, J.-B.; Mulzer, J.; Himmelbauer, M. K. An Approach Toward the Bridged 14Membered Carbon Macrocycle of Bielschowskysin. Eur. J. Org. Chem. 2016, 2016, 2793-2801. (1) Nesic, M.; Kincanon, M. M.; Ryffel, D. B.; Sarlah, D. A new approach towards the synthesis of bielschowskysin: Synthesis and photochemistry of an advanced macrocyclic enedione intermediate. Tetrahedron 2020, 131318.

(16) Nicolaou, K. C.; Adsool, V. A.; Hale, C. R. H. An Expedient Synthesis of a Functionalized Core Structure of Bielschowskysin. Angew. Chem. Int. Ed. 2011, 50, 5149-5152.

(17) (a) Li, Y.; Pattenden, G. Photochemical isomerisation studies of rubifolide and bipinnatin J. Unravelling some of the biosynthesis interrelationships between macrocyclic and polycyclic cembranoids found in corals. Tetrahedron Lett. 2011, 52, 3315-3319. (b) Yang, Z.; Li, Y.; Pattenden, G. Synthesis of E-deoxypukalide, and its biomimetic conversion into deoxypseudopterolide by photochemical ring contraction involving a 1,3-allylic shift. Tetrahedron 2010, 66, 6546-6549.

(18) Refer to the Supporting Information for complete experimental details on isolation, characterization and chemical synthesis.

(19) For an early report on the conformational analysis of Z-configured FBC-macrocycles, see: Marshall, J. A.; Van Devender, E. A. Synthesis of (-)-Deoxypukalide, the Enantiomer of a Degradation Product of the Furanocembranolide Pukalide. J. Org. Chem. 2001, 66, 80378041. A crystal structure of ent-deoxypukalide is also freely accessible from the Cambridge Crystallographic Data Centre (CCDC): CCDC1240121 (link).
(20) Still, W. C. ( \pm )-Periplanone-B. Total synthesis and structure of the sex excitant pheromone of the American cockroach. J. Am. Chem. Soc. 1979, 101, 2493-2495.

(21) Montalvo, D.; Amade, P.; Funel-Le Bon, C.; Fernández, R.; Reyes, F. Acerolide, a new cytotoxic cembranolide from the soft coral Pseudopterogorgia acerosa. Nat. Prod. Res. 2006, 20, 548-552.

(22) Missakian, M. G.; Burreson, B. J.; Scheuer, P. J. Pukalide, a furanocembranolide from the soft coral Sinularia abrupta. Tetrahedron 1975, 31, 2513-2515.

(23) (a) Over, B.; Matsson, P.; Tyrchan, C.; Artursson, P.; Doak, B. C.; Foley, M. A.; Hilgendorf, C.; Johnston, S. E.; Lee, M. D.; Lewis, R. J.; McCarren, P.; Muncipinto, G.; Norinder, U.; Perry, M. W. D.; Duvall, J. R.; Kihlberg, J. Structural and conformational determinants of macrocycle cell permeability. Nat. Chem. Biol. 2016, 12, 1065-1074. (b) Magpusao, A. N.; Rutledge, K.; Hamlin, T. A.; Lawrence, J.-M.; Mercado, B. Q.; Leadbeater, N. E.; Peczuh, M. W. Rules of Macrocycle Topology: A [13]-Macrodilactone Case Study. Chem. Eur. J. 2016, 22, 6001-6011.

(24) For recent reviews on the conformational reorganization of macrocycles, see: (a) Yudin, A. K. Macrocycles: lessons from the distant past, recent developments, and future directions. Chem. Sci. 2015 , 6, 30-49. (b) For a recent review, see: Appavoo, S. D.; Huh, S.; Diaz, D. B.; Yudin, A. K. Conformational Control of Macrocycles by Remote Structural Modification. Chem. Rev. 2019, 119, 9724-9752.

(25) (a) Tinto, W. F.; Chan, W. R.; Reynolds, W. F.; McLean, S. Tobagolide, a new pseudopterane diterpenoid of the octocoral pseudopterogorgia acerosa. Tetrahedron Lett. 1990, 31, 465-468. (b) Rodríguez, A. D.; Soto, J. J. Pseudopterane and Norcembrane Diterpenoids from the Caribbean Sea Plume Pseudopterogorgia acerosa. $J$. Nat. Prod. 1998, 61, 401-404.

(26) To limit the formation of the pseudopterane kallolide A methyl ether 11, the reaction time for the photoirradiation of Z-10 must be short, and the reaction monitored frequently by ${ }^{1} \mathrm{H}$ NMR. In our hands, above 10 mins of irradiation lead to $\mathbf{1 1}$ in up to $12 \%$ isolated yield.

(27) Weinstabl, H.; Gaich, T.; Mulzer, J. Application of the Rodriguez-Pattenden Photo-Ring Contraction: Total Synthesis and Configurational Reassignment of 11-Gorgiacerol and 11-Epigorgiacerol. Org. Lett. 2012, 14, 2834-2837.

(28) (a) Rodriguez, A. D.; Shi, J.-G. The First Cembrane-Pseudopterane Cycloisomerization. J. Org. Chem. 1998, 63, 420-421. (b) Rodriguez, A. D.; Shi, J.-G.; Huang, S. D. Pinnatins A-E: Marine Diterpenes of the Rare Gersolane Class Derived from a Photochemically Induced Rearrangement of a Conjugated 2,5-Bridged Furanocembrane Precursor. J. Org. Chem. 1998, 63, 4425-4432.

(29) Bandurraga, M. M. Natural Product Studies of Selected East Pacific Gorgonians. Ph.D. Dissertation, University of California, San Diego, CA, 1981.

(30) (a) Venkateswarlu, Y.; Sridevi, K. V.; Rao, M. R. New Furanocembranoid Diterpenes from the Soft Coral Sinularia maxima. J. Nat. Prod. 1999, 62, 756-758. (b) Kamel, H. N.; Ferreira, D.; Garcia-Fernandez, L. F.; Slattery, M. Cytotoxic Diterpenoids from the Hybrid Soft Coral Sinularia maxima $\times$ Sinularia polydactyla. J. Nat. Prod. 2007, 70, 1223-1227. (c) Thao, N. P.; Nam, N. H.; Cuong, N. X.; Quang, T. H.; Tung, P. T.; Tai, B. H.; Luyen, B. T. T.; Chae, D.; Kim, S.; Koh, Y.-S.; Kiem, P. V.; Minh, C. V.; Kim, Y. H. Diterpenoids from the Soft Coral Sinularia maxima and Their Inhibitory Effects on Lipopolysaccharide-Stimulated Production of Pro-inflammatory Cytokines in Bone Marrow-Derived Dendritic Cells. Chem. Pharm. Bull. 2012, 60, 15811589. (d) Yang, B.; Liao, S.; Lin, X.; Wang, J.; Liu, J.; Zhou, X.; Yang, X.; Liu, Y. New Sinularianin Sesquiterpenes from Soft Coral Sinularia sp. Mar. Drugs 2013, 11, 4741-4750. (e) Chitturi, B. R.; Tatipamula, V. B.; Dokuburra, C. B.; Mangamuri, U. K.; Tuniki, V. R.; Kalivendi, S. V.; Bunce, R. A.; Yenamandra, V., Pambanolides A-C from the South Indian soft coral Sinularia inelegans. Tetrahedron 2016, 72, 1933-1940. 
(31) (a) Abramson, S. N.; Fenical, W.; Taylor, P. Lophotoxins: irreversible active-site-directed inhibitors of nicotinic acetylcholine receptors. Drug Dev. Res. 1991, 24, 297-312. (b) Abramson, S. N.; Trischman, J. A.; Tapiolas, D. M.; Harold, E. E.; Fenical, W.; Taylor, P. Structure/activity and molecular modeling studies of the lophotoxin family of irreversible nicotinic receptor antagonists. J. Med. Chem. 1991, 34, 1798-804. (c) Rodríguez, A. D.; Shi, J.-G.; Shi, Y.-P. Isolation, Structural Characterization, and Synthesis of a Naturally Occurring Bisfuranopseudopterane Ether: Biskallolide A. Evidence for a Carbocation Intermediate during the Facile Conversion of Kallolide A and Isokallolide A into Various Solvolysis Products. J. Org. Chem. 2000, 65, 3192-3199.

(32) (a) Li, Y.; Palframan, M. J.; Pattenden, G.; Winne, J. M. A strategy towards the synthesis of plumarellide based on biosynthesis speculation, featuring a transannular $4+2$ type cyclisation from a cembranoid furanoxonium ion intermediate. Tetrahedron 2014, 70, 72297240. (b) Lygo, B.; Palframan, M. J.; Pattenden, G. Investigation of transannular cycloaddition reactions involving furanoxonium ions using DFT calculations. Implications for the origin of plumarellide and rameswaralide and related polycyclic metabolites isolated from corals. Org. Biomol. Chem. 2014, 12, 7270-7278.

(33) Doroh, B.; Sulikowski, G. A. Progress toward the Total Synthesis of Bielschowskysin: A Stereoselective [2+2] Photocycloaddition. Org. Lett. 2006, 8, 903-906.

(34) Tang, B.; Simion, R.; Paton, R. S. Thermal and Photochemical Mechanisms for Cyclobutane Formation in Bielschowskysin Biosynthesis. Synlett 2015, 26, 501-507.

(35) Poplata, S.; Tröster, A.; Zou, Y.-Q.; Bach, T. Recent Advances in the Synthesis of Cyclobutanes by Olefin [2+2] Photocycloaddition Reactions. Chem. Rev. 2016, 116, 9748-9815.

(36) Rayner, C. M.; Astles, P. C.; Paquette, L. A. Total synthesis of furanocembranolides. 2. Macrocyclization studies culminating in the synthesis of a dihydropseudopterolide and gorgiacerone. Related furanocembranolide interconversions. J. Am. Chem. Soc. 1992, 114 , 3926-3936.

(37) Chen, R.; Shen, Y.; Yang, S.; Zhang, Y. Conformational Design Principles in Total Synthesis. Angew. Chem. Int. Ed. 2020, 59, 14198. 